Cahiers
de a Recherche
surles Droits
Fondamentaux

Cahiers de la recherche sur les droits fondamentaux

18 | 2020

La vulnérabilité

\title{
Les vulnérabilités numériques
}

Thibault Douville, Chloé Hervochon, Élodie Noël et Yann Paquier

\section{OpenEdition}

Journals

Édition électronique

URL : https://journals.openedition.org/crdf/6462

DOI : $10.4000 /$ crdf.6462

ISSN : 2264-1246

Éditeur

Presses universitaires de Caen

Édition imprimée

Date de publication : 19 novembre 2020

Pagination : 111-119

ISBN : 978-2-84133-987-7

ISSN : 1634-8842

Référence électronique

Thibault Douville, Chloé Hervochon, Élodie Noël et Yann Paquier, « Les vulnérabilités numériques », Cahiers de la recherche sur les droits fondamentaux [En ligne], 18 | 2020, mis en ligne le 19 novembre 2021, consulté le 14 novembre 2022. URL : http://journals.openedition.org/crdf/6462 ; DOI : https:// doi.org/10.4000/crdf.6462 


\title{
Les vulnérabilités numériques
}

\author{
Thibault DOUVILLE \\ Professeur de droit privé à l'université de Caen Normandie \\ Institut Demolombe (EA 967) \\ Chloé HERVOCHON \\ Doctorante en droit privé à l'université de Caen Normandie \\ Institut Demolombe (EA 967) \\ Élodie NOËL \\ Doctorante en droit privé à l'université de Caen Normandie \\ Institut Demolombe (EA 967)

\section{Yann PAQUIER} \\ Doctorant en droit public à l'université de Caen Normandie \\ Centre de recherche sur les droits fondamentaux et les évolutions du droit (CRDFED, EA 2132)
}

I. Les vulnérabilités entre plateformes en ligne et entreprises utilisatrices
A. La définition de la plateforme
B. Les obligations des plateformes

II. La vulnérabilité des personnes concernées par un traitement de données à caractère personnel

A. Le traitement illicite des données personnelles

B. Cybermenaces à l'encontre des données personnelles

III. Les vulnérabilités résultant d'une prise de décision automatisée

A. La vulnérabilité induite par la technique

B. La vulnérabilité induite par le recours aux traitements algorithmiques dans le cadre de l'action administrative: les décisions administratives individuelles

Le développement des technologies numériques suscite autant d'espoirs que de craintes. Des espoirs car elles pourront certainement apporter des remèdes à certaines altérations physiques ou psychologiques. Des craintes aussi car elles bouleversent notre rapport au monde. Laissons de côté les vulnérabilités informatiques et intéressons-nous aux vulnérabilités personnelles, tenant à l'état de la personne, et aux vulnérabilités relationnelles, s'inscrivant dans le rapport aux autres et résultant du pouvoir, notamment économique, exercé sur elle. Celles-ci sont rarement envisagées à travers le prisme de l'environnement numérique.

De manière évidente, l'environnement numérique renforce certaines vulnérabilités. Le législateur en a conscience, il renforce alors le régime protecteur applicable. C'est le cas des mineurs en raison de leur activité en ligne: ils bénéficient d'un droit à l'effacement des données. Cette solution aurait pu être étendue aux majeurs protégés, mais il n'en 
est rien. Dans le domaine économique, la concentration du commerce électronique sur les plateformes en ligne qui ont une double activité de commercialisation directe de produits et de services et d'intermédiation crée des situations de vulnérabilités: les entreprises utilisatrices de plateformes d'intermédiation sont en situation de dépendance à l'égard de ces dernières, ce qui a conduit à l'adoption d'un règlement européen $a d h^{1}$.

Mais l'environnement numérique crée aussi de nouvelles vulnérabilités. Tous vulnérables dans l'environnement numérique? Cette approche est certainement excessive même si l'économie numérique repose sur l'économie de l'attention qui exploite «les biais cognitifs des utilisateurs $»^{2}$. L'exclusion numérique - et donc sociale, économique ou administrative - en est un nouveau type mais tout en contribuant à en accentuer d'autres. Elle touche de nombreuses personnes. Certes l'accès à Internet est rattaché au droit au logement et à la lutte contre la pauvreté3. De même, l'accessibilité des services en ligne aux personnes atteintes d'un handicap est prévue ${ }^{4}$. Mais la maîtrise des technologies numériques est insuffisante. Et le consentement (ou précisément le refus) de la personne concernée est souvent la seule protection dont elle dispose quant à l'utilisation des communications électroniques ${ }^{5}$. Le législateur en a pris conscience, notamment dans le secteur «financier». Ainsi, une exigence de vérification de l'aptitude des clients d'entreprises d'assurance ou d'établissements de crédit à utiliser les communications électroniques a été instaurée à la charge de ces derniers. En retour, les clients se voient accorder le droit de revenir aux échanges sur support papier à tout moment. Toujours est-il qu'une politique d'inclusion numérique forte est nécessaire qui va bien au-delà des initiatives actuelles ${ }^{6}$.

Et que dire aussi de la multiplication des traitements de données à caractère personnel ? Le droit pour les personnes concernées de maîtriser leurs données à caractère personnel demeure limité tandis que l'effectivité de leur protection est discutée ${ }^{7}$. Le recours à des algorithmes décisionnels fait craindre des discriminations en raison de biais, risque accru en présence d'algorithmes autoapprenants. En dehors des hypothèses où ils sont exclus, les garanties prévues semblent bien limitées tant s'agissant de la transparence des algorithmes que de la portée du réexamen de décisions algorithmiques par une personne humaine ${ }^{8}$.
Finalement, les vulnérabilités dans l'environnement numérique sont nombreuses. Elles ne sont pas toujours différentes de celles qui existent dans les rapports sociaux. Toutefois, ces vulnérabilités sont tantôt déformées, amplifiées ou atténuées par le filtre technologique. De même, les technologies numériques sont à l'origine de nouvelles vulnérabilités qui doivent être identifiées. Mais existe-t-il un droit de la vulnérabilité numérique? La réponse est certainement négative, pas plus qu'il existe un droit de la vulnérabilité en général. Mais cela n'interdit pas d'essayer de trouver une cohérence entre les dispositifs protecteurs épars et de souligner les faiblesses de notre droit.

S'inscrivant dans le cadre d'une journée d'étude consacrée au thème "Vulnérabilités et droit, regards croisés", l'étude des vulnérabilités numériques a mobilisé trois jeunes chercheurs privatistes et publicistes spécialisés en droit du numérique. Ils ont cherché à illustrer la manière dont le droit prend en compte et tente de répondre à la vulnérabilité dans l'environnement numérique mais aussi aux situations de vulnérabilité créées par cet environnement. Il en résulte trois études portant sur la situation de vulnérabilité des entreprises utilisatrices à l'égard des plateformes en ligne sur lesquelles elles offrent des biens ou des services (I), sur celle des personnes concernées à l'égard des traitements de leurs données à caractère personnel (II) et enfin sur les vulnérabilités qui résultent des prises de décisions automatisées (III).

\section{Les vulnérabilités entre plateformes en ligne et entreprises utilisatrices ${ }^{9}$}

Alors qu'Internet avait pour objectif premier l'échange et la communication entre deux machines, son utilisation s'est démultipliée avec sa prise en main par les entreprises dont l'idée était de créer des lieux d'échanges entre internautes. Sous l'impulsion de ces grandes entreprises que sont notamment Google ou Amazon, la société est devenue une société où la quête de profits forme la configuration de l'économie. Cette quête repose sur un modèle fondé sur les technologies de l'information, les données et Internet matérialisant un réel enjeu du pouvoir. Ce sont les plateformes numériques, dont les places de marché et moteurs de recherche, qui représentent les nouveaux acteurs prédominant de l'économie. Ces dernières ont profondément

1. Règlement (UE) 2019/1150 du Parlement européen et du Conseil du 20 juin 2019 promouvant l'équité et la transparence pour les entreprises utilisatrices de services d'intermédiation en ligne; voir infra la contribution d'Élodie Noël.

2. C. Zolynski, M. Le Roy, F. Levin, «L'économie de l'attention saisie par le droit», Dalloz IP/IT, 2019, p. 614.

3. Code de l'action sociale et des familles, art. L. 115-3.

4. Par exemple prévoyant que les services de confiance doivent pouvoir, dans la mesure du possible, être accessibles aux personnes atteintes d'un handicap: règlement (UE) 910/2014 du Parlement européen et du Conseil du 23 juillet 2014 sur l'identification électronique et les services de confiance pour les transactions électroniques au sein du marché intérieur et abrogeant la directive 1999/93/CE, art. 15.

5. T. Douville, "Le principe du consentement dans le règlement général sur la protection des données à caractère personnel», in Le règlement général sur la protection des données, A. Bensamoun, B. Bertrand (dir.), Paris, Mare \& Martin, 2020, p. 133 sq.

6. Voir les propositions du Conseil national du numérique dans le rapport L'accessibilité numérique, entre nécessité et opportunité, 5 février 2020 , en ligne: https://cnnumerique.fr/files/uploads/2020/rap-cnnum-accessi-6.pdf.

7. Voir infra la contribution de Chloé Hervochon.

8. Voir infra la contribution de Yann Paquier.

9. Par Élodie Noël. 
modifié les pratiques commerciales mises en œuvre par les acteurs traditionnels. Gain de temps, recherche de prix bas, disponibilité et diversité de l'offre sont les nouvelles attentes auxquelles les plateformes ont su répondre. Ces " puissants facilitateurs de l'entrepreneuriat et de l'innovation" sont devenus les nouveaux intermédiaires du numérique $^{10}$. Ces derniers ne sont pas sans susciter des inquiétudes quant à leurs pratiques commerciales bien souvent abusives, créant un «déséquilibre significatif ${ }^{11}$, manquant de transparence. Tel a été l'objet du litige ${ }^{12}$ relatif à Amazon caractérisant certaines clauses abusives ${ }^{13}$. C'est aux questions posées par ces pratiques commerciales devenues usuelles que le législateur européen a apporté des réponses par le règlement "Platform to Business" $\left(\mathrm{P}_{2} \mathrm{~B}\right) \mathrm{du} 20$ juin $2019^{14}$.

Dans quelles mesures les nouvelles technologies repensent-elles les pratiques commerciales traditionnelles mais surtout en quoi les règles mises en place par le règlement sont-elles suffisantes pour appréhender cette évolution?

S'il est indéniable que les pratiques de ces acteurs représentent une opportunité, le règlement semble être novateur à deux égards, l'un tendant à la consécration d'une définition de la plateforme (A) l'autre en leur imposant une pluralité d'obligations (B).

\section{A. La définition de la plateforme}

De par leurs pratiques, les plateformes sont devenues "des sources de vulnérabilité ${ }^{15}$ pour l'ensemble de la société. En effet, les services d'intermédiation en ligne ont pris une place «considérable dans les échanges commerciaux mondiaux $»^{16}$ et sont matérialisés par les moteurs de recherche, les places de marché. C'est avant tout leur position d'intermédiaire et leur poids sur le marché qui a été la clé de voûte de ce règlement. C'est parce qu'elles remettent en cause les canons du droit déjà bien établis qu'elles représentent un enjeu majeur. Aussi, le règlement est novateur en ce qu'il donne une définition fonctionnelle des plateformes. Au sens du présent règlement, un service d'intermédiation en ligne est un service répondant à trois conditions cumulatives: un service répondant à la définition de service de la société de l'information au sens de la directive de $2015^{17}$, c'est-à-dire «tout service presté normalement contre rémunération, à distance, par voie électronique et à la demande individuelle d'un destinataire de services $»^{18}$; un service permettant "aux entreprises utilisatrices d'offrir des biens ou services aux consommateurs ${ }^{19}$ et ayant pour mission de faciliter les transactions; un service fourni «sur la base de relations contractuelles entre le fournisseur de ces services et les entreprises utilisatrices ${ }^{20}$.

Au regard de ces différents éléments, plusieurs constats peuvent être faits.

Le règlement vient compléter la loi pour une République numérique ${ }^{21}$ en ce qu'il appréhende les relations avec les entreprises et non seulement les relations avec les consommateurs non professionnels.

Le législateur européen a, pour la première fois, consacré une définition de la plateforme d'intermédiation en ligne, prenant conscience de manière effective de leur rôle majeur dans l'économie. Ce règlement marque les prémices d'une rupture avec la vision désuète de certaines plateformes au sens de la directive dite « commerce électronique ${ }^{22}$ du 8 juin 20oo. En effet, cette dernière permettait aux plateformes ayant un rôle neutre, «en ce que [leur] comportement est purement technique, automatique et passif, impliquant l'absence de connaissance ou de contrôle des données qu' [elles] stockent » ${ }^{23}$, d'être qualifiées d'intermédiaires techniques bénéficiant d'une responsabilité extrêmement limitée. Or, les plateformes «avaient toujours revendiqué le bénéfice ${ }^{24}$ de cette directive, profitant de l'appréhension par le droit quasi inexistante des nouvelles technologies. Le règlement $\mathrm{P}_{2} \mathrm{~B}$ permet de pallier en partie cette dérive en prenant en compte le caractère spécifique des plateformes mais surtout leur fonction première qu'est l'intermédiation en ligne. C'est avant tout à des fins d'unicité des règles applicables aux plateformes en ligne pour l'ensemble de l'Europe que les institutions européennes ont mis en œuvre ce règlement, évitant une multiplicité des législations en la matière.

10. A. Cousin, «Plateformes d'intermédiation: les nouvelles règles européennes», Expertises, n 452, 2019, p. 391.

11. Au sens de l'article L. 442-6 du Code de commerce.

12. TC Paris, 2 septembre 2019, $\mathrm{n}^{\circ} 2017050625$.

13. Sur ce point, voir A. Lecourt, "Sanction des pratiques commerciales abusives d'Amazon», Dalloz IP/IT, 2019, p. 710; H. Barbier, «Le statut juridique de la plateforme en ligne: intermédiaire contractuel et non simple support technique», Revue trimestrielle de droit civil, 2019 , p. 848.

14. Règlement (UE) 2019/1150.

15. V.-L. Benabou, L. Cytermann, C. Zolynski, «Bilan de l'Agenda numérique européen: quand la poussière retombe», Revue de l'Union européenne, 2020 , p. 15.

16. A. Cousin, «Plateformes d'intermédiation...», p. 391.

17. Directive (UE) 2015/1535 du Parlement européen et du Conseil du 9 septembre 2015 prévoyant une procédure d'information dans le domaine des réglementations techniques et des règles relatives aux services de la société de l'information.

18. Ibid., art. $1^{\mathrm{er}}, \S 1$, pt b.

19. Règlement (UE) 2019/1150, art. 2, \$2, pt b.

20. Ibid., art. $2, \$ 2$, pt c.

21. Loi nº 2016-1321 du 7 octobre 2016 pour une République numérique.

22. Directive 2000/31/CE du Parlement européen et du Conseil du 8 juin 2000 relative à certains aspects juridiques des services de la société de l'information, et notamment du commerce électronique, dans le marché intérieur.

23. CJUE, GC, 23 mars 2010, Google France SARL c. Louis Vuitton Malletier SA et autres, C-236/o8 à C-238/o8, \$ 114

24. V.-L. Benabou, L. Cytermann, C. Zolynski, «Bilan de l'Agenda numérique européen...», p. 15. 
Cependant, loin d'unifier, c'est un enchevêtrement législatif qui a été créé, opacifiant d'autant plus la mise en œuvre de la responsabilité des plateformes. En principe, la plateforme dont l'activité repose uniquement sur la mise en relation, le stockage, doit être qualifiée d'intermédiaire technique, sauf à démontrer qu'elle exerce une influence déterminante dans la réalisation de la prestation fournie par son intermédiaire. Si la plateforme propose plusieurs services et s'ils sont indissociables, la qualification de l'ensemble dépendra de la qualification de la prestation principale. Tel a été le cas de la société Uber dans un arrêt de la CJUE de $2017^{25}$. Il en ressort deux éléments, la mise en œuvre d'une qualification unitaire du service et une qualification dépendant de l'identification de «l'élément principal ${ }^{26}$ du service. La prestation principale d'Uber étant le service de transport et non un service de la société de l'information, les deux étant indissociables, c'est la qualification de prestation de transport qui l'emporte. A contrario, si les services de la plateforme sont dissociables, c'est-à-dire indépendants économiquement, alors une responsabilité distributive pourra être mise en œuvre, encore faut-il que leurs régimes respectifs ne soient pas incompatibles.

Or le règlement $\mathrm{P}_{2} \mathrm{~B}$ n'appréhende que le service d'intermédiation en ligne éludant le caractère protéiforme des plateformes. En effet, la plupart offrent un service de stockage, mettent en relation et offrent leurs propres services en concurrence directe avec les entreprises utilisatrices de cette plateforme.

En outre, un devoir de diligence étant mis à la charge des plateformes, se pose la question de leur réelle neutralité à l'égard des contenus mis en ligne par leur intermédiaire et donc de l'adéquation avec la directive sur le commerce électronique. En effet, comment pouvoir faire preuve de transparence et de vigilance tout en état neutre et ne pas avoir connaissance des contenus stockés?

\section{B. Les obligations des plateformes}

Outre une définition de fournisseur de service d'intermédiation en ligne, c'est un objectif de responsabilisation ainsi que de transparence de leurs pratiques commerciales qui a été poursuivi par le législateur. Ce dernier impose une obligation de clarté dans l'établissement des conditions générales d'utilisation des plateformes ${ }^{27}$ et plus largement un droit au référencement.
Afin de mettre en échec le déséquilibre significatif imposé par les plateformes, une obligation de transparence et plus généralement d'information repose sur les plateformes, ces dernières devant préciser de manière " claire et compréhensible ${ }^{28}$ l'ensemble des informations insérées dans les conditions générales d'utilisation mais aussi les critères de classement des entreprises utilisatrices. Dans la lignée de l'obligation de loyauté imposée par la loi pour une République numérique ${ }^{29}$ à l'égard des consommateurs non professionnels, le règlement reconnaît une obligation d'information précontractuelle et contractuelle à l'égard des entreprises utilisatrices. La mise en œuvre de cette obligation permet de répondre aux "préoccupations quant aux pouvoirs des plateformes ${ }^{30}$. À l'heure où la plateforme publicitaire Google détient $85 \%$ des parts de marché depuis $2006^{31}$, comment endiguer un anéantissement de la concurrence si ce n'est en pouvant vérifier leurs pratiques. En effet, le classement opéré par un fournisseur de service d'intermédiation en ligne «a une incidence importante sur le choix des consommateurs" et donc "sur la réussite commerciale des entreprises utilisatrices ${ }^{32}$. Si, en principe, le classement doit être opéré de manière impartiale, certaines plateformes dont Google et Amazon ont été épinglées en raison de l'imprécision des critères de classement et du favoritisme à l'égard de leurs propres services. Le moteur de recherche Google référençait en priorité son site comparateur Google Shopping ${ }^{33}$ au détriment des autres alors qu'Amazon faisait preuve d'un manque de transparence et de clarté quant aux critères de référencement des entreprises ${ }^{34}$. Dès lors, en imposant la communication des critères de classement, de leur opportunité pour le consommateur, du caractère payant ou non, le législateur tend à garantir le bon fonctionnement du marché et une concurrence pérenne.

Pour autant, cette volonté de transparence doit être tempérée, l'équilibre voulu par le législateur ne reposant que sur un fil. En effet, si les fournisseurs ont l'obligation d'indiquer leurs paramètres de fonctionnement, rien ne leur impose «de divulguer les algorithmes ${ }^{35}$ ou plus largement l'ensemble des informations relevant des secrets d'affaires. Or, tout l'intérêt d'un moteur de recherche réside dans la performance de son algorithme et de ses calculs permettant de classer les résultats en fonction de leur pertinence. Aussi, comment allier transparence et conservation du fonctionnement de l'algorithme puisque

25. CJUE, GC, 20 décembre 2017, Asociación Profesional Elite Taxi c. Uber Systems Spain SL, C-434/15.

26. T. Douville, "Arrêt Uber ou l'art délicat de la qualification", La semaine juridique, entreprise et affaires, $\mathrm{n}^{\circ}$ 10, 8 mars $2018,1111$.

27. Règlement (UE) 2019/1150, art. 3.

28. Ibid.

29. Code de la consommation, art. L. 111-7.

30. V.-L. Benabou, L. Cytermann, C. Zolynski, «Bilan de l’Agenda numérique européen... », p. 15.

31. Sur ce point, voir F. Fatah, «La souveraineté à l'ère du numérique: enjeux stratégiques pour l'État français et les institutions européennes», Revue de l'Union européenne, 2020, p. 26.

32. Règlement (UE) 2019/1150, cons. 24.

33. Commission européenne, résumé de la décision du 27 juin 2017 relative à une procédure d'application de l'article 102 du traité sur le fonctionnement de l'Union européenne et de l'article 54 de l'accord EEE, Journal officiel de l'Union européenne, C 9, p. 11.

34. TC Paris, $1^{\text {re }}$ ch., 2 septembre 2019, RG 2017050625.

35. Règlement (UE) 2019/1150, art. 6 . 
c'est précisément grâce à lui que le classement est opéré laissant une échappatoire pour les fournisseurs de services d'intermédiation en ligne.

Par conséquent, si le règlement reste une avancée majeure, la mise en place d'un corpus législatif favorisant l'émergence des services en ligne a occasionné une évolution fulgurante du nombre des plateformes leur donnant la possibilité d'asseoir leurs pratiques dans un contexte d'absence de régulation. En définitive, «les comportements déloyaux des géants de l'Internet ${ }^{36}$ sont tels que le législateur a aujourd'hui laissé place à une volonté protectrice tant du consommateur que des entreprises utilisatrices.

\section{La vulnérabilité des personnes concernées par un traitement de données à caractère personnel ${ }^{37}$}

En 2017, selon la Commission européenne ${ }^{38}$, les données représentaient 272 milliards d'euros. Les données personnelles, anciennes données nominatives, sont les informations qui se rapportent à une personne physique identifiée ou identifiable, directement ou indirectement: la personne concernée. À l'ère du big data, c'est-à-dire de la possibilité de récupérer rapidement une multitude de données, ces dernières sont collectées et traitées en masse pour des finalités diverses. Quel est l'intérêt et l'enjeu de la collecte de ces données? Lucratif avant tout. Elles permettent de connaître les centres d'intérêt et les besoins d'une personne, d'influencer son comportement d'achat et elles peuvent être revendues à prix d'or sur le darkweb. Plus effrayant encore, elles peuvent être croisées pour déduire d'autres informations sur l'individu, influencer son comportement ou être utilisées pour prendre des décisions à son égard. Face à l'enjeu majeur de protection de ces données et les vulnérabilités toujours plus nombreuses, le législateur européen a encadré leur collecte et leur traitement. Depuis le 25 mai 2018 c'est le RGPD ${ }^{39}$ qui est applicable.

Il s'agit alors de s'interroger sur les vulnérabilités auxquelles doivent faire face les données personnelles. Elles sont principalement de deux types: en premier lieu, la donnée peut faire l'objet d'un traitement illicite, à l'insu des personnes concernées, et, dans un second temps, le sens qu'elle contient peut être révélé ou atteint. Concernant le traitement illicite, outre le fait que la personne perde le contrôle sur ses données personnelles, ces finalités peuvent être dangereuses notamment si elles permettent le profilage de l'individu, à savoir, prédire son comportement, ses préférences, sa santé, etc., et prendre des décisions le concernant. En outre, les données personnelles contiennent une information, elles ont un sens qui les rend vulnérables en cas d'événements susceptibles d'entraîner une violation de sécurité: les cybermenaces. Ainsi, la personne concernée est touchée directement par ces vulnérabilités puisqu'il est question de la protection de sa vie privée et de ses données personnelles.

Quelles sont les solutions proposées par le législateur? Ces solutions sont-elles suffisantes et permettent-elles une protection efficace? Il s'agit d'envisager ces problématiques concernant le traitement illicite des données personnelles (A) et les cybermenaces qui pèsent sur elles (B).

\section{A. Le traitement illicite des données personnelles}

Il faut donc s’interroger sur les vulnérabilités liées à un traitement illicite des données personnelles, c'est-à-dire un traitement réalisé à l'insu de la personne concernée, sans respecter les principes relatifs au traitement l'empêchant d'exercer un contrôle sur ses données et risquant d'engendrer des discriminations. Ce traitement peut alors permettre de surveiller son mode de vie et / ou d'effectuer un profilage pour anticiper ses actions, ses préférences, voire pour prendre des décisions à son égard. Des affaires célèbres ont d'ailleurs démontré la dangerosité de tels traitements, comme Prism et la surveillance de millions de personnes ou Cambridge analytica qui via le profilage d'individus a permis d'influencer des élections. Le profilage présente des risques pour les droits et libertés des personnes et notamment pour leur vie privée.

Face à ces enjeux, le législateur européen est intervenu avec le RGPD pour réglementer l'utilisation des données personnelles et éviter les dérives. Pour que le traitement soit licite, et sauf exception, il est nécessaire que la personne concernée y consente de manière non équivoque ${ }^{40}$. Le traitement doit être licite, loyal et transparent et la collecte de données doit avoir des finalités déterminées et limitées à ce qui est nécessaire ${ }^{41}$. De plus, le traitement est, en principe, interdit sur les données sensibles révélant des informations telles que l'origine raciale ou ethnique, les opinions politiques ou encore les convictions religieuses de la personne.

L'objectif est de protéger la personne concernée et des prérogatives lui ont même été accordées puisque le responsable du traitement doit lui transmettre des

36. M. Behar-Touchais, "Les pratiques commerciales déloyales des plateformes liées à la publicité sur Internet», in Rôle et responsabilité des opérateurs de plateforme en ligne: approche(s) transversale(s) ou approches sectorielles?, J. Sénéchal, S. Stalla-Bourdilon (dir.), Paris, IRJS, 2018, p. 146.

37. Par Chloé Hervochon.

38. Commission européenne, «Créer une économie européenne fondée sur les données», COM(2017) 9 final, 10 janvier 2017 , p. 2.

39. Règlement (UE) 2016/679 du Parlement européen et du Conseil du 27 avril 2016 relatif à la protection des personnes physiques à l'égard du traitement des données à caractère personnel et à la libre circulation de ces données, et abrogeant la directive 95/46/CE (Règlement général sur la protection des données - RGPD).

40. RGPD, art. 6

41. RGPD, art. 5 . 
informations telles que les finalités de ce traitement, la durée de conservation de ces données, l'existence de ses droits et la présence d'un éventuel profilage ${ }^{42}$. Par la suite, la personne peut obtenir de la part du responsable de traitement un accès à ses données afin de savoir si elles sont traitées ou non ${ }^{43}$. Elle peut également demander la rectification, l'effacement de ses données ${ }^{44}$ ou la limitation du traitement ${ }^{45}$ et elle peut même $s^{\prime} y$ opposer ${ }^{46}$ à tout moment.

Les traitements illicites existent malgré tout et il convient de se pencher sur les sanctions prévues. Tout d'abord des amendes administratives peuvent être prononcées par la Commission nationale informatique et libertés (la CNIL, qui est l'autorité de contrôle en France) et elles peuvent aller jusqu'à $4 \%$ du chiffre d'affaires de l'entreprise ou 20 millions d'euros en cas de non-respect des règles édictées par le RGPD. Il y a également des sanctions pénales pour les traitements qui ne respectent pas la réglementation avec des peines maximales de cinq ans d'emprisonnement et 300 ooo euros d'amende. Sont réprimés la collecte de données personnelles par des moyens frauduleux, déloyaux ou illicites ${ }^{47}$, la collecte malgré l'opposition de la personne concernée ${ }^{48}$, la conservation de données sensibles ${ }^{49}$ ou de données personnelles pour une durée supérieure à la durée initiale ${ }^{50}$ et le détournement des finalités du traitement ${ }^{51}$.

Tous ces mécanismes sont-ils suffisants? Malheureusement non, puisqu'une difficulté majeure persiste: la personne concernée accepte volontairement le traitement de ses données personnelles en contrepartie de la gratuité du service. Vous connaissez l'adage en numérique qui affirme que, si c'est gratuit, c'est que vous êtes le produit. L'individu communique sciemment une multitude d'informations lorsqu'il utilise les réseaux sociaux, lorsqu'il s'inscrit sur des sites Internet mais également via les objets connectés qu'il possède, et leur nombre explose (ordinateurs, smartphones, montres, enceintes ou aspirateurs connectés). Toutes les données que l'on consent à transmettre sont analysées et traitées. Il y a un paradoxe puisque les individus commencent à réaliser la vulnérabilité de leurs données personnelles mais leur comportement demeure imprudent. La vigilance de la personne concernée est pourtant la clé d'une meilleure protection de ses données et il faut réussir à lui en faire prendre conscience. Ainsi, la pédagogie et la sensibili- sation semblent être indispensables pour anticiper et limiter de nombreux risques.

En dehors du traitement illicite, les risques de violation de sécurité des données personnelles sont omniprésents aujourd'hui.

\section{B. Cybermenaces à l'encontre des données personnelles}

Il s'agit alors de s'interroger sur les cybermenaces pesant sur les données personnelles et nous pouvons en recenser trois types: l'atteinte à leur intégrité, l'atteinte à leur confidentialité et l'atteinte à leur disponibilité. Plusieurs personnes vont être touchées par la violation des données personnelles: la personne concernée puisque ses droits seront atteints et le responsable du traitement qui va perdre la confiance de ses utilisateurs et subir de multiples pertes financières. Par exemple, en 2017, une agence de crédit américaine s'est fait pirater provoquant une fuite des données sensibles de ses clients et donc une atteinte à leur vie privée mais, dans le même temps, une perte de valeur des actions de la société de $35 \%$ en une semaine ${ }^{52}$.

L'atteinte à l'intégrité de la donnée personnelle est une altération, une modification non autorisée. Ces dernières sont alors corrompues, elles ne correspondent plus à la réalité. Par exemple, un pirate informatique qui pénètre le système d'une université pour modifier les notes des étudiants.

L'atteinte à la confidentialité résulte d'un accès non autorisé ou d'une divulgation non autorisée de ces données. Il s'agit principalement des fuites ou des vols de données personnelles qui sont particulièrement répandues. Par exemple, entre le 25 mai et le $1^{\text {er }}$ octobre 2018 , la CNIL a reçu 742 signalements de fuites de données correspondant aux données de 33,7 millions de personnes ${ }^{53}$, mais on peut également citer Yahoo en 2013 avec 3 milliards de comptes de ses utilisateurs touchés ou Uber en 2016 avec les données de 57 millions d'utilisateurs.

Pour finir, l'atteinte à la disponibilité des données est une destruction ou une perte des données personnelles. Elle peut résulter de la perte d'un périphérique (ordinateur, clé USB) contenant des données personnelles ou encore de leur chiffrement comme la cyberattaque dont a été victime le CHU de Rouen. Cette attaque est un rançongiciel (ou

2. RGPD, art. 13 et 14 .

43. RGPD, art. 15 .

44. RGPD, art. 17

45. RGPD, art. 18

46. RGPD, art. 21.

47. Code pénal, art. 226-18.

48. Code pénal, art. 226-18-1.

49. Code pénal, art. 226-19.

50. Code pénal, art. 226-20.

51. Code pénal, art. 226-21.

52. Le club des juristes, Assurer le risque cyber, janvier 2018, p. 31, rapport disponible en ligne: https://www.leclubdesjuristes.com/wp-content/ uploads/2018/o1/cdj_rapport_cyber-risk_janvier-2018_fr_web.pdf.

53. CNIL, «Violations de données personnelles: $1^{\text {er }}$ bilan après l'entrée en application du RGPD», 16 octobre 2018, en ligne: https://www.cnil.fr/fr/ violations-de-donnees-personnelles-1er-bilan-apres-lentree-en-application-du-rgpd. 
ransomware en anglais), un logiciel malveillant chiffrant l'intégralité des données du système contre le paiement d'une rançon pour récupérer la clé de déchiffrement. Au CHU de Rouen, les données médicales des patients qui sont des données personnelles ont été chiffrées.

Afin de lutter contre ces vulnérabilités, le RGPD a prévu diverses obligations à la charge du responsable du traitement qui doit anticiper les violations par la sécurisation. Il est ainsi tenu de mettre en œuvre les mesures techniques et organisationnelles appropriées afin de garantir un niveau de sécurité adapté au risque ${ }^{54}$. Pour ce faire, il doit sécuriser le traitement afin d'empêcher ou du moins limiter les violations de données. Pour les traitements les plus à risque, il doit réaliser une analyse d'impact du traitement sur la protection des données ${ }^{55}$.

Il y a donc des garanties de sécurité afin d'assurer l'intégrité et la confidentialité des données. Malgré tout, des violations peuvent avoir lieu et le responsable de traitement doit alors notifier l'incident à la CNIL dans les 72 heures $^{56}$ si la violation peut engendrer un risque pour les droits et libertés des personnes physiques. Il doit également informer la personne concernée si l'incident présente des risques importants pour ses droits et libertés ${ }^{57}$. Si le responsable du traitement n'a pas sécurisé son traitement ou n'a pas notifié l'incident subi, il peut être sanctionné par une amende administrative de la CNIL ou par une sanction pénale pouvant aller jusqu'à cinq ans d'emprisonnement et 300000 euros d'amende ${ }^{58}$.

Le cybercriminel risque les mêmes peines puisque l'atteinte à l'intégrité ou à la disponibilité des données ${ }^{59}$ et la divulgation sans autorisation de données personnelles ${ }^{60}$ sont sanctionnées pénalement.

L'intérêt de la sécurité du traitement est multiple mais elle est notamment essentielle en ce qu'elle entraîne la confiance. Des données personnelles traitées sans sécurisation présentent un danger important pour la vie privée de la personne concernée. Plusieurs difficultés apparaissent pour garantir une sécurité effective des données personnelles: l'ingéniosité des cybercriminels avec des techniques de piratage en constante mutation et l'interconnexion des systèmes qui favorise la propagation des atteintes au niveau mondial. En effet, les experts en sécurité doivent se former constamment et effectuer des veilles sur l'état de la menace afin de s'armer contre les cybercriminels. Néanmoins, l'interconnexion des systèmes permet aux virus de se diffuser rapidement à l'échelle mondiale, comme le démontrent les rançongiciels Wannacry et Notpetya et leurs conséquences dommageables à travers le monde. Pour conclure, une législation européenne est donc une première étape pour contrer ces vulnérabilités mais, au sein du cyberespace sans frontière, elle semble insuffisante...

\section{Les vulnérabilités résultant $d^{\prime}$ une prise de décision automatisée $e^{61}$}

Le recours aux traitements algorithmiques et leur multiplication engendrent des situations de vulnérabilité, pouvant aller jusqu'à remettre en cause certains droits et libertés. Nous entendrons par vulnérabilité « toute fragilité morale ou matérielle, individuelle ou sociale à laquelle une personne se trouve exposée ${ }^{62}$. Pour illustrer ce problème émergent, des sources de vulnérabilités technologiques peuvent être induites par le recours à la technique (A). Puis, nous aborderons les décisions administratives individuelles fondées sur les traitements algorithmiques car elles s'imposent parfois en toute opacité malgré l'absence de consentement des administrés, du fait de prérogatives de puissances publiques, ajoutant à la vulnérabilité technique une vulnérabilité juridique (B).

\section{A. La vulnérabilité induite par la technique}

Un algorithme est un «ensemble de règles opératoires dont l'application permet de résoudre un problème énoncé au moyen d'un nombre fini d'opérations " ${ }^{63}$. Il va faire l'objet d'une intégration dans un code informatique pour être exécuté: l'intérêt est d'obtenir l'automatisation de la résolution du problème. Rappelons qu'ils ne sont pas neutres ${ }^{64}$.

Qu'il s'agisse d'algorithmes auto-apprenants ${ }^{65} \mathrm{ou}$ moins sophistiqués, ils peuvent contenir des biais. D'une part, les biais algorithmiques peuvent résulter d'un souci de modélisation du problème et de sa résolution, d'autre part, de nombreux biais résultent des données scrutées par les algorithmes. Ces deux points peuvent engendrer

60. Code pénal, art. 226-22.

61. Par Yann Paquier.

62. F.-X. Roux-Demare, «La notion de vulnérabilité, approche juridique d'un concept polymorphe», Les cahiers de la justice, $\mathrm{n}^{\circ} 4,2019$, p. 620.

63. Dictionnaire Larousse en ligne, "Algorithme», https://www.larousse.fr/dictionnaires/francais/algorithme/2238.

64. "Il est en effet vain de demander aux algorithmes d'être "neutres" alors qu'ils sont généralement conçus pour choisir, trier, filtrer ou ordonner les informations selon certains principes» (D. Cardon, "Le pouvoir des algorithmes», Pouvoirs, n 164, janvier 2018, La datacratie, p. 65).

65. Selon le Conseil constitutionnel, les algorithmes auto-apprenants sont «susceptibles de réviser eux-mêmes les règles» en fonction des données qu'ils analysent (CC, déc. $\mathrm{n}^{\circ}$ 2018-765 DC du 12 juin 2018, Loi relative à la protection des données personnelles, $\left.\$ 66\right)$. 
des discriminations ${ }^{66}$, ou des outils d'aide à la décision trompeurs pour les utilisateurs. En effet, comme toute personne, les concepteurs sont influencés aussi bien par l'environnement social, leurs expériences personnelles que par l'état de l'art dans leur domaine.

Au début de la crise du Covid-19, des chercheurs de l'INSERM ont proposé un outil d'aide à la décision publique pour estimer le risque de son importation en Europe ${ }^{67}$, et qui considérait que les pays à risque étaient le Royaume-Uni et l'Allemagne. Bien sûr, cet outil n'était pas présenté comme ayant un but prédictif, mais comme " un outil théorique d'aide à la décision publique » ${ }^{68}$. Or, cette simulation n'incluait que des données parcellaires sur le trafic aérien venant de République populaire de Chine, ne tenant pas compte des liens étroits entre certains pays et le Hubei, berceau de l'épidémie. Il ne s'agit pas de remettre en cause ces outils, mais de les appréhender avec mesure pour éviter que les gouvernants, et donc l'État, ne soient victimes de programmes mal conçus.

Les algorithmes auto-apprenants augmentent le risque de biais, surtout quand leur apprentissage n'est pas supervisé par des humains. Ces technologies, bien que prometteuses, permettent d'obtenir des critères inférés à partir des données et non plus juste en fonction de critères voulus par les concepteurs. Le risque est donc de voir reproduire des violations du droit positif car les données d'apprentissage comporteraient des biais exacerbés par l'outil. Telles les activités bancaires d'Apple gérées aux États-Unis par la banque Goldman Sachs où les femmes se voyaient octroyer un crédit inférieur aux hommes «alors qu'ils ont les mêmes conditions fiscales et le même historique de crédit » ${ }^{69}$.

Indépendamment des biais, les logiciels peuvent renfermer des fraudes. Un éditeur américain a reconnu avoir favorisé les intérêts d'une société pharmaceutique dédiée aux opioïdes par un outil d'aide à la prise de décision recommandant aux médecins leur prescription ${ }^{70}$. Le risque de vulnérabilité peut alors concerner le patient comme le professionnel de santé qui parfois ne comprend plus le fonctionnement des recommandations.

Enfin, il ne faut pas négliger l'ordinateur qui exécute le programme, dont les composants peuvent dysfonc- tionner et ainsi contredire la volonté des développeurs. Par exemple, quand les logiciels font leur immixtion dans des domaines sensibles tels les scrutins nationaux ${ }^{71}$, la vulnérabilité pourrait être conséquente. Le corps électoral est vulnérable par rapport aux machines à voter, ce qui peut en cas de dysfonctionnement altérer la sincérité du scrutin, et donc remettre en cause la volonté réelle des électeurs.

\section{B. La vulnérabilité induite par le recours aux traitements algorithmiques dans le cadre de l'action administrative: les décisions administratives individuelles}

Le constitutionnaliste Lawrence Lessig est célèbre pour son adage: "Code is law» $»^{72}$. Mais parfois, il s'agit de «Law is code» : il est possible d'implémenter dans un programme les règles de droit que l'administration souhaiterait voir appliquer.

L'ancien article 10 de la loi informatique et libertés de 1978 disposait qu'

Aucune $[. .$.$] décision produisant des effets juridiques à$ l'égard d'une personne ne peut être prise sur le seul fondement d'un traitement automatisé de données destiné à définir le profil de l'intéressé ou à évaluer certains aspects de sa personnalité ${ }^{33}$.

Il n'était donc pas interdit pour l'administration de fonder des décisions administratives individuelles sur de tels traitements dès lors qu'il y avait intervention humaine ${ }^{74}$.

La source de vulnérabilité réside toutefois dans l'application de règles non prévues par le droit positif à cause des vulnérabilités techniques vues, et qui influencerait ensuite l'agent dans sa prise de décision. Cette situation est d'autant plus problématique quand l'algorithme est secret, «dès lors que celui qui l'a créé ou le met en œuvre ne le communique pas $»^{75}$. Il était urgent d'assurer une transparence de ces dispositifs opaques. Le législateur, dès $2016^{76}$, sous l'impulsion de la doctrine de la Commission d'accès aux documents administratifs (CADA) ${ }^{77}$ et du juge

66. En ce sens, voir J. Charpenet, C. Lequesne Roth, «Discrimination et biais genrés, les lacunes juridiques de l'audit algorithmique», Recueil Dalloz, 2019, p. 1852-1857.

67. INSERM, "Coronavirus: des chercheurs de l'INSERM proposent un modèle pour estimer le risque d'importation de l'épidémie en Europe ", 24 janvier 2020, en ligne: https://presse.inserm.fr/coronavirus-des-chercheurs-de-linserm-proposent-un-modele-pour-estimer-le-risquedimportation-de-lepidemie-en-europe/380oo.

68. Ibid.

69. A. Jean, "Quand les algorithmes discriminent les femmes", Le Point, 23 novembre 2019, en ligne: https://www.lepoint.fr/invites-du-point/ aurelie-jean-quand-les-algorithmes-discriminent-les-femmes-23-11-2019-2349124_420.php.

70. Department of Justice of the United States, «Electronic Health Records Vendor to Pay \$145 Million to Resolve Criminal and Civil Investigations", 27 janvier 2020, en ligne: https://www.justice.gov/opa/pr/electronic-health-records-vendor-pay-145-million-resolve-criminal-and-civil-investigations-o.

71. F. Pellegrini, "Chaînes de confiance et périmètres de certification: le cas des systèmes de "vote électronique" ", rapport de recherche, RR-8553, INRIA, 2014, en ligne: https://hal.inria.fr/hal-o1010950v4/document.

72. L. Lessig, «Code Is Law: On Liberty in Cyberspace», Harvard Magazine, janvier-février 2000.

73. Loi $\mathrm{n}^{\circ} 78-17$ du 6 janvier 1978 modifiée par la loi nº 2004-801 du 6 août 2004, art. 10.

74. Par exemple, voir CNIL, décision $n^{\circ}$ MED-2017-053 du 30 août 2017 mettant en demeure le ministère de l'Enseignement supérieur, de la recherche et de l'innovation.

75. T. Douville, «Parcoursup et le secret des algorithmes», Dalloz IP/IT, 2019, p. 700.

76. Loi $\mathrm{n}^{\circ}$ 2016-1321 du 7 octobre 2016 pour une République numérique.

77. En ce sens, voir CADA, avis nº 20161989 du 23 juin 2016. 
administratif ${ }^{78}$, a assimilé les codes sources à des documents administratifs communicables ${ }^{79}$, sauf quand sont en jeu des secrets et intérêts protégés par la loi ${ }^{80}$. Malgré leur communication, ils sont difficiles à comprendre pour les profanes.

Le législateur a donc prévu des dispositions spécifiques pour les administrés ayant fait l'objet d'une décision individuelle prise sur le fondement d'un traitement algorithmique, notamment car les traitements automatisés ne portent pas toujours sur des données à caractère personnel ${ }^{81}$. Ces décisions doivent mentionner explicitement qu'un tel traitement est survenu, afin que l'intéressé (personne physique ou morale) puisse demander, même si la décision est favorable, que lui soient communiquées de manière intelligible les principales caractéristiques du programme ${ }^{82}$. Cependant, cette avancée importante pour la transparence se heurte à des exceptions juridiques ${ }^{83}$.

Le législateur ${ }^{84}$ a finalement autorisé le recours aux décisions administratives fondées exclusivement sur un traitement algorithmique dès lors que le traitement ne porte pas sur des données à caractère personnel sensibles ${ }^{85}$. Mais l'on imagine la crainte suscitée par ces outils pouvant sans intervention humaine produire des effets de droit intéressant les tiers, tout en faisant des erreurs, voire créant de nouvelles règles de droit pour les algorithmes auto-apprenants.

Le Conseil constitutionnel, dans une décision du 12 juin $2018^{86}$, s'est prononcé sur la conformité des décisions individuelles exclusivement automatisées. Il rappelle qu'elles doivent avoir la mention explicite prévue à l'article L. 311-3-1 du CRPA et qu'aucun secret ou intérêt protégé par la loi ne peut être opposé à la communication des principales caractéristiques du traitement ${ }^{87}$. De plus, ces décisions doivent pouvoir faire l'objet d'un recours administratif ou contentieux. Pour le premier, l'administration doit pouvoir se prononcer sans recours à un traitement automatisé. En cas de contentieux contre la décision, le juge peut exiger la communication des caractéristiques de l'algorithme ${ }^{88}$. Quant au responsable de traitement, il doit pouvoir expliquer à l'intéressé de manière intelligible son fonctionnement. Pour cela, il doit maîtriser le traitement et ses évolutions. Pour les algorithmes auto-apprenants, le Conseil ajoute que, «sans le contrôle et la validation du responsable du traitement", ils ne peuvent fonder exclusivement une décision administrative individuelle ${ }^{89}$. En l'état de l'art en informatique, cela revient à dire qu'ils doivent être exclus car la transparence du processus décisionnel ne peut être totale. Le Conseil est alors venu préciser un régime juridique pour faire cesser une vulnérabilité juridique sur ces algorithmes.

Enfin, à partir du $1^{\text {er }}$ juillet 2020 , les décisions administratives individuelles reposant exclusivement sur de tels traitements comportent, à peine de nullité, la mention explicite prévue à l'article L. 311-3-1 du CRPA $^{90}$.

78. TA Paris, 10 mars 2016, M. X, nº 1508951.

79. Voir Code des relations entre le public et l'administration (CRPA), art. L. 30o-2. Et, sous condition, les administrations doivent également publier en ligne les « règles définissant les principaux traitements algorithmiques utilisés dans l'accomplissement de leurs missions lorsqu'ils fondent des décisions individuelles» (CRPA, art. L. 312-1-3 et D. 312-1-4).

80. Ils ne peuvent donc être communiqués que dans les conditions prévues par le livre III du CRPA, et à l'exception de l'art. L. 612-3 du Code de l'éducation. Voir supra.

81. En effet, la loi n ${ }^{\circ}$ 78-17 du 6 janvier 1978 modifiée par l'ordonnance $n^{\circ}$ 2018-1125 du 12 décembre 2018 permet, lorsqu'il y a un traitement de données à caractère personnel, de connaître la logique qui sous-tend le traitement (art. 47).

82. CRPA, art. L. 311-3-1 et R. 311-3-1-1.

83. Tel est le cas par exemple lorsqu'est en jeu un secret de défense nationale. Voir les exceptions prévues à l'article L. $311-5,2^{\circ}$ du CRPA et à l'article L. 612-3 du Code de l'éducation. Lire aussi T. Douville, «Parcoursup et le secret des algorithmes», p. 700-702; T. Douville, «Parcoursup à l'épreuve de la transparence des algorithmes ", Dalloz IP/IT, 2019, p. 390-393.

84. Loi n ${ }^{\circ} 78-17$, art. 47 .

85. Sont considérées comme des données à caractère personnel sensibles les catégories de données listées à l'article 8, I de la loi $\mathrm{n}^{\circ} 78$-17.

86. CC, déc. $\mathrm{n}^{\circ} 2018-765$ DC du 12 juin 2018.

87. Ibid., $\$ 70$.

88. Ibid.

89. Ibid., $\$ 71$.

90. Loi $\mathrm{n}^{\circ} 78-17$, art. $47,2^{\circ}$. 